\title{
Home Treatment bei psychiatrischen Erkrankungen
}

Viele Kinder und Jugendliche in Österreich sind psychisch krank, doch wenige werden adäquat behandelt: Über ein Drittel der 10 bis 18-jährigen leidet einmal in ihrer Jugend an psychischen Erkrankungen, aber nur für die Hälfte von ihnen reichen die altersgerechten Behandlungskapazitäten. Die Behandlung Betroffener im eigenen Zuhause durch entsprechend qualifizierte Personen bietet hier eine Möglichkeit, Behandlungsressourcen zu entlasten und gleichwertige oder sogar bessere Erfolge zu erzielen. Das Austrian Institute for Health Technology Assessment (AIHTA) hat dazu die internationale Evidenz analysiert und nun die Ergebnisse in einer aktuellen Studie veröffentlicht.

\section{Die Ergebnisse im Detail}

In der Studie [1] wurden sechs internationale Modelle für Home-Treatment aus vier Ländern $(3 \times$ aus Deutschland, jeweils $1 \times$ aus den Niederlanden, den USA und Kanada) verglichen. Die Kinder und Jugendlichen in den Modellen waren überwiegend zwischen 5 - 18 Jahre alt, der Großteil der Modelle umfasste alle psychiatrischen Diagnosen und erstreckte sich über eine Behandlungsdauer von drei bis vier Monaten. Die Ergebnisse einer Analyse der Wirksamkeit dieser Modelle waren dann erstaunlich eindeutig, wie Priv. Doz. Dr. Claudia Wild, Direktorin des AIHTA, kommentiert: „Es zeigte sich klar, dass Home-Treatment zu einer Verbesserung der psychopathologischen Symptome der Kinder und Jugendlichen führt. Insbesondere bei psychosozialen Störungen kann diese Behandlungsform einen tollen Beitrag leisten. Wir konnten da sogar feststellen, dass sie stationären Behandlungen in punkto Wirksamkeit überlegen sein kann."
Auch zeigte sich, dass Home-Treatment weniger Krankenhausaufenthalte für die Kinder und Jugendlichen erforderlich machte und diese ggf. von kürzerer Dauer waren. Dazu ergänzt Wild:„Zu beachten gilt es aber auch, dass sich nicht alle psychiatrischen Erkrankungen gleichermaßen für eine Behandlung zu Hause eignen. Auch muss im Vorfeld geklärt werden, ob das familiäre Umfeld den Krankheitsverlauf möglicherweise negativ beeinflussen könnte. Dann wäre eine stationäre Behandlung natürlich von Vorteil".

Basis dieser klaren Ergebnisse war das Erfassen der wissenschaftlichen Nachweise für die Wirksamkeit des Home-Treatments im Rahmen der Modelle. Diese Nachweise fokussierten auf eine veränderte Symptomatik und etwaige Notwendigkeiten für eine Hospitalisierung genauso wie auf die Behandlungszufriedenheit der Betroffenen und den Bedarf an weiteren Behandlungen. Das eindeutige Ergebnis der Auswertung dieser sechs internationalen Modelle und der wissenschaftlichen Evaluationen macht das Home-Treatment in den Augen des AIHTA zu einer echten Alternative oder Ergänzung zur stationären Behandlung.

\section{Auch in Österreich?}

Vor dem Hintergrund dieser Fakten setzt sich das AlHTA gemeinsam mit multidisziplinären Expertinnen und Experten mit Überlegungen zur Implementierung des Home-Treatments in Österreich auseinander. Auch die Ergebnisse dieses Prozesses finden in dem nun veröffentlichten Bericht Eingang und münden in klaren Empfehlungen des AIHTA. Als ganz wesentlich erachtet das Team um Wild dabei die
Koordination eines Home-Treatments mit den zahlreichen, bestehenden kinder- und jugendpsychiatrischen Angeboten in Österreich. Auch müsste das mit dem Home-Treatment betraute Personal entsprechend geschult und die dafür notwendigen Ressourcen eingeplant werden. Um eine evidenzbasierte Entwicklung im Bereich der Kinder- und Jugendpsychiatrie zu fördern, empfiehlt das AIHTA abschlieBend auch eine begleitende Evaluation bei Einführung des Home-Treatments in Österreich.

\section{Literatur \\ 1. Wolf S, Grössmann N (2020) Home- Treatment in der Kinder- und Jugendpsy- chiatrie: Eine Analyse zur Wirksamkeit und möglichen Implementierung in Österreich. HTA-Projektbericht 129. https://bit.ly/ 2Qzyqy5}

\section{Korrespondenzadresse}

Priv. Doz. Dr. phil. Claudia Wild

Austrian Institute for Health Technology Assessment

Garnisongasse 7/20, 1090 Wien, Österreich claudia.wild@aihta.at

Hinweis des Verlags. Der Verlag bleibt in Hinblick auf geografische Zuordnungen und Gebietsbezeichnungen in veröffentlichten Karten und Institutsadressen neutral.

Paediatr. Paedolog. 2021 · 56:139 https://doi.org/10.1007/s00608021-00902-9

(c) Springer-Verlag GmbH Austria, ein Teil von Springer Nature 2021

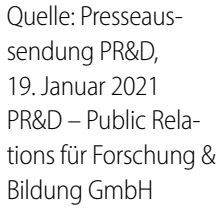

